\title{
A Roadmap Towards the Smart Factory
}

\author{
Amr T. Sufian \\ Faculty of Engineering and Technology \\ Liverpool John Moores University \\ Liverpool, UK \\ a.t.sufian@ljmu.ac.uk \\ Roderick Wah \\ Beverston Engineering Ltd \\ Prescot, UK \\ rod@beverston.co.uk
}

\author{
Badr M. Abdullah \\ Faculty of Engineering and Technology \\ Liverpool John Moores University \\ Liverpool, UK \\ B.M.Abdullah@ljmu.ac.uk \\ David Clements \\ Beverston Engineering Ltd \\ Prescot, UK \\ David.clements@beverston.co.uk
}

\author{
Muhammad Ateeq \\ Faculty of Engineering and Technology \\ Liverpool John Moores University \\ Liverpool, UK \\ m.ateeq@ljmu.ac.uk
}

\begin{abstract}
Industry 4.0 is the transformation of industrial manufacturing through digitisation and the use of different emerging technological advancement, when coupled together forms the smart factory. However, the roadmap of adoption is a journey rather than an absolute solution. The objectives of this paper are to give general insights and a roadmap towards the smart factory. A six-gear roadmap concept is proposed and discussed together with different challenges and practical ways of overcoming them. The significance of this paper can serve as a steppingstone for a detailed strategic roadmap for a successful implementation and transformation into a smart factory.
\end{abstract}

Keywords- Industry4.0, IIoT, Smart Factory, Smart Manufacturing

\section{INTRODUCTION}

The first industrial revolution started in the late $18^{\text {th }}$ century when agricultural societies became industrialised and urban which was driven by steam engines, waterpower and mechanisation. This transition was going from hand methods to machines and moving people from home into purpose-built factories. The second industrial revolution in the late $19^{\text {th }}$ century was another great leap forward in technological advancement mainly driven by electricity which lead to advances in manufacturing and production methods such as the introduction of the assembly line by Henry Ford [1] enabling mass production especially for automobiles and airplanes that revolutionised public transport. The third industrial revolution appeared at the second half of the $20^{\text {th }}$ century with the rise of electronics, computers and information technology led to the automation of production using Programmable Logic Controllers (PLC's) and robotics in manufacturing. The fourth industrial revolution is underway which builds upon the third industrial revolution and the technological advances driven by the advancement of the internet, power of advanced computing, data science, low cost of sensors, and new levels of connectivity leading to a new technological phenomenon called 'digitisation' [2].

This digitalisation enables changes in the manufacturing industry by connecting the digital and the physical world together making factory automation more flexible and offering opportunities for increasing efficiency in production [3]. It enables to build a new virtual world from which the physical world can be steered. It is also interpreted as the application of the cyber physical systems within industrial production systems [4].
The term Industry 4.0 is commonly referred to the fourth industrial revolution. It was introduced by the German government national strategic initiative High Tech 2020 to drive digital manufacturing forward by increasing digitisation and enhancing competitiveness in the manufacturing industry [5]. It has become institutionalised with the platform Industrie 4.0 and the Reference Architectural Model Industrie 4.0 (RAMI 4.0) [6] that now serves a central point of contact for policy makers showing how to approach industry 4.0 in a structural manner. Although Germany was the first to tap into this new way of pursuing digitisation in the manufacturing industry it has become a global trend. Another initiative such as the UK 'Made Smarter' strategy 2030 sets out how the UK manufacturing can be transformed through the adoption of industrial digital technologies to become a global leader in industrial digitisation [7] with the potential to create enormous opportunities for the manufacturing industry by increasing growth, creating new jobs, reducing $\mathrm{CO}_{2}$ emission and improving industrial productivity. The aerospace industry was identified by the Made Smarter review as one of the sectors which has the greatest potential to gain from Industry 4.0 with $£ 17.5$ billion opportunity through adoption of digital technologies that has the potential to reduce cost and build new business models [7]. Other global strategies and initiatives are also in place to support the digital transformation and competitiveness in manufacturing. The Made in China 2025 initiative [8] aims to move manufacturing up the value chain in China. America Makes [9] in the USA supports additive manufacturing and 3D printing. South Korea Manufacturing Innovation 3.0 [10] supports the adoption and transformation into the smart factory.

There are technological advancements in digital industrial technologies that make the trend Industry 4.0, they are considered as the transforming force that will modify the production infrastructure, the development of products \& services and improving customer relationships $[3,11]$. These digital trends are the Industrial Internet of Things (IIoT), Big Data, Artificial Intelligence (AI), Additive Manufacturing, Digital Twin \& Simulation, Advances in Robotics \& Automation (e.g. Collaborative Robots (Cobots)), Argumentative \& Virtual Reality, Cloud Computing and Cyber Security [3].

Industry 4.0 is seen by manufactures as a toolbox of a rich collection of available technologies, therefore it's important to know how to use these tools and where would they create 
value to the business and deliver on the customer requirements [12]. The smart factory concept is an outcome of successful connectivity and integration of key elements of Industry 4.0 technologies that results in connecting people, machines and objects along the entire manufacturing process offering opportunities for increasing efficiency in production, cutting downtime, saving resources and producing less waste $[3,11,13,14]$. Real-time access and connectivity of data from multiple sources in the factory is valuable because it allows manufacturers to utilise this data and respond to the actionable information derived from the data [15]. This allows manufacturers to differentiate themselves from the others by making better decision regarding the manufacturing process, hence the term 'Data Driven Manufacturing' is revolutionary $[14,16]$.

This paper will introduce a six-gear roadmap concept for implementing the smart factory outlining key enabling technologies and highlighting different challenges ahead and practical examples of overcoming them.

\section{A SIX-GEAR ROADMAP}

The transformation into a smart factory is a journey rather than a complete solution and the best way to implement it is through a gradual process and building on initial digitised capabilities. Fig. 1 shows a six-gear roadmap concept inspired from the motoring industry that can serve as a steppingstone for a further detailed strategic roadmap towards a successful implementation and transformation into a world class smart factory. Its divided into six stages starting from having a clear strategy, building seamless connectivity and effective system integration to ways of applying analytics and data science to the manufacturing process data and highlighting further ways of scaling the concept inside and outside the factory.

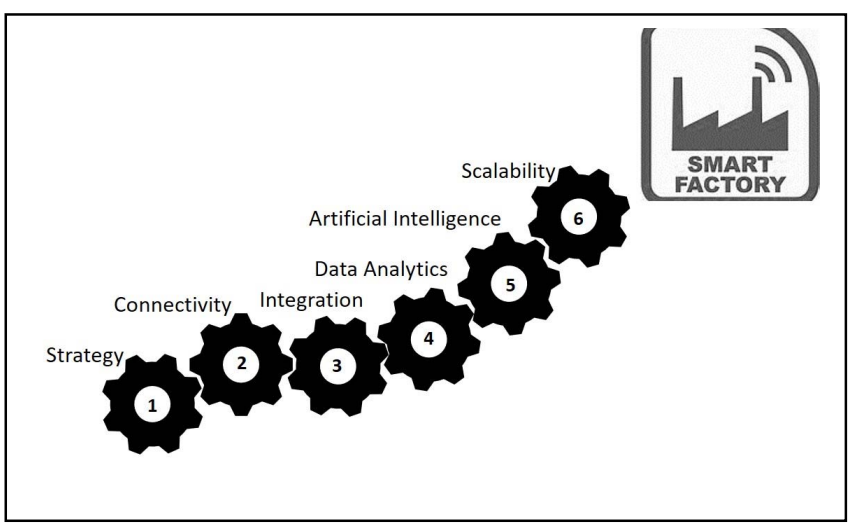

Fig. 1. A Six-Gear Road Map for the Smart Factory Journey

\section{A. Gear 1: Build A Strategy}

Manufacturing companies have started to appreciate and embrace industrial digitisation and the potential it offers to improve efficiencies in production, cost reduction, responsiveness to the market and customer demands [12]. However it can be confusing of how it can be achieved in practice, therefore a strategy has to be in place to guide manufactures with a clear vision and objectives of what they want to achieve out of it in a way that best suits their business model and prioritising the areas in which digitisation would offer the most benefit and a Return On Investment (ROI).
Although manufacturing companies within the same sector might share similar drives (e.g. the aerospace industry share common drives to increase production rate as demand grow, to reduce costs for competitive edge, to add value to the products and services and to maintain and improve quality [17]). The starting point can be different, and the journey can be bespoke, therefore, it is important to firstly get started on the journey, understand where the value is to be created and identify the starting point [17].

There have been considerable efforts by large organisations and solution providers to produce white papers guiding manufacturers with practical tips in their Industry 4.0 digital transformation journey. FESTO ten practical tips [18] and Bosch-Rexroth practical roadmap [12] are good examples, all advising business to implement it through a step-by-step approach and building on initial digitised capabilities.

\section{B. Gear 2: Connectivity}

The internet has become an essential part of our daily lives and there are many Internet of Things (IoT) devices connected with each other over the internet with around five million devices linked and 6.4 billion data-communicating objects-with the number forecasted to increase to 20 billion by 2020 [19]. In industry this has become rapidly important for competitive manufacturing enabling real-time monitoring and access to data insights about manufacturing process.

Information Technology (IT) and Operation Technology (OT) departments within the manufacturing operate independently, the first managed business applications from the front office and the latter kept plant operations running smoothly. IT technologies such as Enterprise Resource Planning (ERP) and Manufacturing Execution System (MES) made both departments work together making more means of the operational data [20]. Industrial Internet of Things (IIoT) builds on current and emerging technologies combining appropriate elements of IT and OT that have the potential to bring plant and business performance to a new level [21]. This is achieved by increasing automation, connectivity and integration in a single networked system that will successfully enable the transformation into smart manufacturing [22].

\section{1) IT Infrastructure}

Collecting real-time data from machines, sensors and production in the shop floor is important for data driven manufacturing. The amount of information generated will increase exponentially and there is a need for fast communication network for acquiring such information in real-time [23]. Building on existing IT Infrastructure capabilities and networks in the shop floor can be the starting point. Expanding further depending on the volume of data generated and communicated through the IT infrastructure, having an on premises solution (e.g. servers) or migration to the cloud or having a bit of both (a hybrid solution), bearing in mind the limitation of efficiency and the security of the data, have to be considered. Although Industrial Ethernet has been the de facto backbone of many plant floor connectivity, It is worth to mention that the emergence of $5 \mathrm{G}$ technologies is seen as a key enabler of next generation manufacturing systems [24] operating at much higher frequencies and larger bandwidth. 


\section{2) IIoT Gateway}

The IIoT gateway combines hardware (sensors fitted onto a cell, machine tool, etc.), software (for collecting and processing data at the edge/fog), communication and networking technologies (Wi-Fi, Bluetooth, 5G, Ethernet, RFID, etc.). All these components must work in coherence to enable communication between the intelligent physical entities in the plant or in the field to each other, to the internetenabled devices, to a digital platform or infrastructure without inferring with any existing automation logic [21].

In the aerospace industry, regular audits, inspections and quality checks are performed during manufacturing and assembly and less dependent on the use of robotics and automation because of the tight regulations regarding aircraft construction therefore, IIoT enabled devices are key to enable workers with tools to increase productivity [11].

\section{3) Reference Architectures:}

There has been several initiatives and close collaboration between research and industry to standardise reference architectures for the IIoT connectivity in industrial applications, which they all serve as a general guide and aim to facilitate interoperability, simplicity and ease of development [25]. Reference architectures like the Industrial Internet Reference Architecture (IIRA) and the Industrial Internet Connectivity Framework (IICF) by the Industrial Internet Consortium (IIC) provide broad industry application to help evaluate connectivity of IIoT solution [26, 27]. The Internet of Things-Architecture (IoT-A) provides detailed overview of the IoT information technology aspects [28]. RAMI 4.0 provides a three-dimensional map for smart factories covering manufacturing and logistics applications [29]. The International Organization for Standardization (ISO) and the International Electrotechnical Commission (IEC) provides six domain model frameworks for the (IoT) Reference Architecture [30] focusing on resilience, safety and security.

\section{4) Cyber Security (CS)}

Cyber Security (CS) is one of the main challenges to the connectivity of the smart factory. As soon as an IIoT device is connected to the internet, if not handled correctly, makes the whole network less secure and resilient, therefore CS becomes critical because of the risks associated with it. An example of such risks can be hacking the machines causing the production process to stop or even causing danger to the operator if the machines are externally manipulated. Also, as manufacturing networks grow, so does the attack surface and to protect critical manufacturing assets, a cyber-security strategy defence must be in place to address the threats.

To get started on building a CS strategy, risks have to be identified because security measures can be defined based on the risk profiles of the connected devices [31]. In manufacturing most likely the risks will be the machines, tools and the data they handle, its functions within the network operation and the potential impact to the network when it's breached. Once the risks have been identified, security strategies can be implemented where applicable from designing a secure IIoT infrastructure, installation and the configuration of the connected devices and managing and maintaining such infrastructure should all be considered. There are regulations and code of practices available that one can start from in order to identify suitable procedures and measures ensuring privacy and security of connected devices over the internet. The Network Information Systems Directive (NIS) [32], General Data Protection Regulations (GDPR) [33] and Secure by Design Initiative (SDI) developed by the National Cyber Security Centre (NCSC) and Information Commission Office (ICO) [34] provides the art of developing robust set of processes in order to maintain a secure network of connected devices ensuring solid foundation for Industry 4.0 CS strategies. Another reference architecture such as ISO/IEC 30141 [30] focuses on the integration of multiplicity of devices and operations within IoT for the system to perform safely and securely when faced with distributions such as natural disasters, faults, humans' errors and attacks. The Industrial Internet Security Framework (IISF) [35] published by IIC provides framework for system designers to identify procedures that should be applied for addressing security relevant to IIoT systems. In industrial manufacturing, the confidentiality or privacy of the data collected from the plant floor is not crucial because of no personal data is involved therefore, security measures to protect such data that complies with the General Data Protection Regulations (GDPR) [33] might not be required. However, the integrity of the data collected is fundamental to the operation of the service and that has to comply with the NIS Directive [32] as IIoT connected devices provide data collection service to the manufacturing industry.

In general, when designing the smart factory connectivity architecture, a starting point can be getting the IT departments on board from the start to assist on building upon existing IT security policies, processors and procedures to address the inclusion of connected devices and their interoperability [13]. A good example of an industrial collaboration to address CS in the smart factory connectivity architecture was the iSMART factory concept [36] between global machine equipment manufacturers "MAZAK" and the IT giants "Cisco" teaming up together to connect machines securely via MTConnect protocols and Industrial IT switches which has embedded network security to help prevent any issues with unauthorised access to or from the machines and equipment within the network.

Once the IT Infrastructure, IIoT connectivity architecture and CS strategies are laid out, the low hanging fruit data can be collected from the physical entities in the plant floor for real time condition monitoring.

\section{Gear 3: System Integration}

ERP systems play a major role connecting day-to-day activities across the entire manufacturing facility providing essential insights into operations and manufacturing process (materials/inventory/production/operation/accounting) integrated into one database [37]. Beside ERP systems other common IT based platforms that are used in the manufacturing industry are Manufacturing Execution System (MES), Supply Chain Management (SCM), Product Lifecycle Management (PLM), etc. may need to be connected and integrated within the smart factory architecture connectivity making it one of the challenging applications and cornerstones of Industry 4.0 vision [23, 38, 39]. Production and work in progress data in the factory are 
normally monitored via MES embedded in substations across the plant acting as a bridge between the plant floor and the office ERP systems. However most MES systems are fully dependent on the operator input and are not based on real time data, therefore IIoT and MES needs to be connected and integrated with each other to synchronise machines data with the production data while keeping the operator input to a minimum level (e.g. logging quality issues). This will allow greater insights into the production process, making faster decisions for corrective actions and by correlating it with ERP systems should optimises production process, work orders and other resources needs which can have significant improvements in business and financial performance [40]

Recent reports have shown that only few digital advanced manufacturing companies have fully integrated and correlated plant data with enterprise applications since a more advanced connectivity and integration are needed [38]. To address the integration and connectivity challenges between different OT and IT systems, the use of open standards and protocols are becoming more essential [40]. The Service Oriented Architecture (SOA) approach (e.g. Http, REST, etc.), open Application Programming Interface (API) connectivity along with the combination of smart and cost effective devices as Human Machine Interface (HMI) to allow a two-way directional flow of information would assist and simplify the integration and connectivity $[23,40]$.

Table 1 shows a list of leading IIoT platforms \& systems produced by leaders in the field that use best practices to facilitate different types of protocols, open standards and architectures for connectivity and integration between IIoT and enterprise applications bridging the gap between IT and OT. These platforms don't replace or rebuild long established systems but acts as a new layer to the infrastructure orchestrating the new way of industry 4.0 by sourcing data from anywhere with minimal connectivity and integration cost and disruption together with having analytical capabilities to deliver insights via dashboards, mobile devices or even augmented reality display.

TABLE I.: List of leading IIoT platforms

\begin{tabular}{|l|l|l|}
\hline Platform Name & Company & General Features \\
\hline ThingWorx & PTC & IIoT-C, DA, AI \\
\hline MindSphere & Siemens & IIoT-C, DA, AI, CB \\
\hline Watson IoT & IBM & IIoT-C, DA, AI \\
\hline Nexceed (PPM) & BOSCH & IIoT-C, DA \\
\hline Kinetic & Cisco & IIoT-C, DA \\
\hline WISE-PasS & Advantech & IIoT-C, DA, AI, CB \\
\hline Cumulocity IoT & Software AG & IIoT-C, DA, AI, CB \\
\hline AWS IoT & Amazon Web Services & IIoT-C, DA, AI, CB \\
\hline Predix & GE Digital & IIoT-C, DA, AI, CB \\
\hline Azure IoT & Microsoft & IIoT-C, DA, AI, CB \\
\hline FactoryWiz & FactoryWiz & IIoT-C, DA, MES \\
\hline machinemetrics & MachineMetrics & IIoT-C, DA, MES, AI, CB \\
\hline ForcamForce & FORCAM & IIoT-C, DA, MES \\
\hline
\end{tabular}

(IIoT-C: IIoT Connectivity; DA: Data Analytics; Artificial Intelligence; MES: Manufacturing Execution System; ERP: Enterprise Resources Planning, CB: Cloud Based)

\section{Gear 4: Real-Time Data Analytics}

One of the main components of Industry 4.0 is data analytics based on larger amount of data collected from different sources (sensor, machines, IT based systems, etc.) [3]. The resulting vast amounts of data has become associated with the term 'Big Data' due to the volume, variety, velocity, variability and value [41]. Big data becomes smart data when collected in a structured manner and condensed turning the data into actionable information used for decision making. Amongst the most common key performance indicators (KPI's) among manufacturers gauging the health of their business are; the Overall Equipment Effectiveness (OEE), on-time-delivery, average manufacturing cycle time, downtime vs. operating time, first pass yield and other financial performance indicators such as net profit margins, means cost per unit etc. [42]. Data analytics create value in manufacturing by providing a diagnostic approach enabling root-cause problem solving and guide with solutions to reduce deviations that occur during manufacturing.

The OEE is commonly used in the factory [43] which is a type of KPI that combines different sort of data from different systems (e.g. machines, MES and ERP) enabling deep-dive analytics to be achieved based on three key factory parameters, (Availability, Performance and Quality) allowing to effectively evaluate the manufacturing operation. IIoT connectivity combined with an integrated IIoT platform would facilitate the automation of data collection, data exchange, and analytics in real time in order to optimise and increase OEE to provide management with greater visibility for improved decision making.

One of the key challenges in dealing with big data is complexity, therefore cleansing, handling, time stamping and initial processing of the data at the edge (IIoT gateway) are needed before transporting it to the fog (cloud or on-premises data centre) for more detailed analytics in order to reduce the complexity, volume and network bandwidth [44].

\section{E. Gear 5: Artficial Intellegence (AI)}

Artificial Intelligence (AI) from an industrial point of view means algorithm-based and data-driven computer-based systems that enhance machines and people with digital capabilities such as perception, reasoning, learning and even autonomous decision-making [45]. In the manufacturing industry AI is seen as the brain of the digital transformation while other tools provide the muscle [46] driving this transition from automation to autonomy. A survey constructed by Hewlett Packard Enterprise (HPE) with European industrial companies expect AI to contribute in improving efficiencies in operations, maintenance, supply chain, cutting down the costs and increasing revenue streams while improving customer experience by enhancing products, services and adding new features [47]. Another survey by International Data Cooperation (IDC) predicts that manufactures will rely on AI platforms in the future to drive digital transformation across the supply chain and network related products and assets into digital twin ecosystems [48].

Predictive maintenance is one of the key values created by applying AI to the data to spot trends in machine data in order to give early warnings and indications of possible failures and breakdown allowing maintenance to be scheduled and 
intervened rather than periodic maintenance enabling proactive behaviours rather than reactive. Digital twining is a digital model approach which is used to digitally simulate machines, production lines and even the whole factory in different configurations based upon the historical data collected and data mining procedures. This enables virtual verification and validation of the different scenarios of the manufacturing process in order to assess current manufacturing capabilities, optimising operations and to give early discovery of the system performance and deficiencies before the physical processes are developed [4, 49]. For the aerospace manufacturing industry, the value is created in using digital twin as a tool for zero defects manufacturing in order to reduce the level of waste and quality issues associated with new product development and maintaining the current demand for high quality standards. Autonomous implementation of rules and methods in manufacturing can also be achieved using AI to further recommend or act to address raised issues making machines to adjust themselves to optimise quality or energy efficiency during production [50]. However, data is the main challenge for AI as the lack of data and data quality to feed AI modules could stumble from AI adoption, therefore a strategy must be in place for AI adoption. Such strategies can be started from identifying use cases, selecting relevant data mining procedures and using robust platforms that will be able to compute and provide adequate visualisation [47].

Other key challenges of adopting AI is that it's often seen as it will take jobs away. However, according to HPE survey [47], AI-created jobs will balance or outweigh these made redundant by AI. Other challenges are the lack of analytical skills and knowledge, therefore the development of internal staff and hiring external expertise can possibly overcome that.

\section{F. Gear 6: Scalability}

The smart factory concept is not only for condition monitoring of machines in order to increase productivity of the factory but it's also about looking for ways to optimise this productivity based on data collected and shared from inside and outside the factory. Scaling the use of IIoT gateways across the plant and linking it up with the smart factory network infrastructure could give deep insights and assist different ways to optimise productivity. Environmental condition monitoring is a good example of scaling the use of IIoT in the plant floor in order to assess the relationship between productivity of machines/people with the environmental conditions, the data could possibly suggest ways on how to best optimise and increase productivity. Integration of the supply chain with IIoT connectivity and AI providing information insights into every part of the supply chain will enable awareness, visibility, responsiveness and resilience giving the manufacturers enormous opportunities to capitalise on in order to stay competitive and overcome challenges like meeting customer demands of tighter deadlines and increasing cost pressures [51].

\section{OTHER CHALLANGES}

\section{A. The Cost of Implmentation}

The cost of the implementation of Industry 4.0 technologies in factories are always a primary concern for SME manufacturers since limited data is available to demonstrate the ROI to justify the affordability as well as the cost of training/hiring staff [14]. Government funded schemes are key to support and encourage manufacturers to adopt Industry 4.0 technologies. The UK Made Smarter program [52] is an example of a leading scheme in the North West of England designed to boost manufacturing productivity and growth across the region with dedicated programs and co-funding opportunities to assist the digital transformation.

\section{B. Managing Change}

Industry 4.0 is a significant digital transformation project that is associated with change, therefore resistance is expected because people do often resist procedures outside the norm. Understanding and accepting change and effectively managing that change within the organisation is key to the successful transformation into a smart factory. Manufacturing companies need to get the buy in from the whole organisation by addressing their concerns (e.g. fear of unknown, no personal reward, job security, etc.), having stakeholders on board, building communication lines and establishing cross functional teams will all help to equip manufacturing organisation for this change [18].

\section{Uplifting Skills}

Increasing automation, data analytics, AI and robotics are forcing workforce in production facilities to change how they work from ordinary shop-floor tasks to allowing them to spend more time on higher-value activities such as monitoring datasets for preventative maintenance etc. [7]. Manufacturing organisations must be prepared to support this development making sure that workforce has the required level of technical skills, together with other soft skills such as creativity, critical thinking and communication skills in order to help existing employees get up to speed with new technologies and recruiting people who already have required skills and expertise $[53,15]$.

\section{CONCLOUSION}

- Improving productivity and efficiency in manufacturing requires working smarter using smart tools.

- Technological advancement in industry 4.0 technologies provides the tools when coupled together allows seamless connectivity, effective system integration and more automation in manufacturing providing real-time data analytics and deeper insights for better decision making regarding the manufacturing process.

- Becoming a smart factory is a journey and requires a roadmap to guide the manufacturing industry through its adaptation.

- A six-gear roadmap concept is proposed and discussed with strategies and examples to overcome the different challenges along the way which could assist a successful implementation and transformation into a smart factory.

\section{ACKNOWLEDGMENT}

This work was supported by Beverston Engineering Ltd \& the Knowledge Transfer Network under grant no. 011153.

\section{REFERENCES}

[1] S. Watts, The People's Tycoon-Henry Ford and the American Century. New York: Vintage Books, 2006. 
[2] W. Schroeder, "Germany's Industry 4.0 strategy, Rhine capitalism in the age of digitisation" Friedrich-Ebert-Stiftung (FES), FES London

November 2016, Available: https://www.feslondon.org/fileadmin/user_upload/publications/files/FES London_Schroeder_Germanys-Industrie-40-Strategy.pdf.

[3] M. Rüßmann, M. Lorenz, P. Gerbert, M. Waldner, J. Justus, P. Engel and M. Harnisch, "Industry 4.0: The Future of Productivity and Growth in Manufacturing Industries", 9 April 2015, Available: https://www.bcg.com/publications/2015/engineered products project bus iness_industry_4_future_productivity_growth_manufacturing_industries.a spx.

[4] M. Ghobakhloo, "The future of manufacturing industry: a strategic roadmap toward industry 4.0", Journal of Manufacturing Technology Management, vol. 29 , no. 6 , p. $36,2018$.

[5] D. Klitou, J. Conrads, M. Rasmussen, CARSA and Laurent Probst \& Bertrand Pedersen, PwC "Germany Industrie 4.0", European Commission Directorate, Digital Transformation Monitor January 2017, Available: https://ec.europa.eu/growth/tools-

databases/dem/monitor/content/germany-industrie-40.

[6] Platform Industrie 4.0, "Digitization of Industrie Platform Industrie 4.0", Federal Ministry for Economic Affairs and Energy (BMWi), Berlin 2016, Available: https://www.bmwi.de/Redaktion/EN/Publikationen/digitizationof-Industrie.html.

[7] (2017). Made Smarter Review. Available: https://www.gov.uk/government/publications/made-smarter-review

[8] S. Kennedy, "Made in China 2025", 1/6/2015. Accessed on: 22/05/2019, Available: https://www.csis.org/analysis/made-china-2025

[9] (22/05/2019). America Makes. [Online] Available: https://www.americamakes.us/about/.

[10] J. E. Ha, "Smart Industry Korea", Netherlands Enterprise Agency, 27 September 2015, [Online]. https://www.rvo.nl/sites/default/files/2015/10/Smart\%20Industry\%20in\%2 0Korea.pdf.

[11] V. Bonneau, B. Copigneaux, IDATE, L. Probst, B. Pedersen, O. Lonkeu, PwC "Industry 4.0 in Aeronautics: IoT applications", European Commission Directorate, Digital Transformation Monitor, June 2017, [Online]. Available: https://ec.europa.eu/growth/toolsdatabases/dem/monitor/content/industry-40-aeronautics-iot-applications.

[12] A. Minturn, M. Lomax, P. Streatfield "A practical roadmap for the implantation of Industry 4.0", in "Industry 4.0 White Paper", Bosch Rexroth Group, 2018, [Online]. Available: https://www.boschrexroth.com/en/gb/trends-and-topics/industry-40/industry-4-0-white-paper/industry-4-0-white-paper-1.

[13] T. Gurela. (2018, 1 February) Industry 4.0 and the factory network. I4.0 today Available: http://i40today.com/magazines/

[14] J. Wójcicki. (2018, 16 February) Industry 4.0: The Future of Smart $\begin{array}{llll}\text { Manufacturing. } & \text { Industry } & 4.0 & \text { Available: }\end{array}$ https://www.industry40summit.com/latest-news/magazine

[15] N. Kampe, "Technology in Industry Report", Automation Alley 2018, Available: https://automationalley.com/techreport

[16] M. Albert, "Plain Talk about Data-Driven Manufacturing", ed: Modern Machine Shop, 2016

[17] S. Savani. (2018, August) Industry Focus- Aerospace- How organisations in the sector can capitalise on industry 4.0. Industry 4.0 Available: https://www.industry40summit.com/latest-news/magazine

[18] FESTO, "Practical tips for Industry 4.0 implementation", in "Industry 4.0" FESTO, 2019, [Online]. Available: https://www.festo.com/cms/en$\mathrm{gb} \mathrm{gb/65731.htm,} \mathrm{Accessed} \mathrm{on:} \mathrm{03/03/2019}$

[19] E. Schaeffer, Industry X. 0: Realizing digital value in industrial sectors. Kogan Page Publishers, 2017.

[20] Cisco, The Ultimate Guide to Smart Manufacturing: Cisco, 2018. [Online]. Available: https://www.cisco.com/c/dam/m/digital/elqcmcglobal/OCA/Assets/manufacturing/Cisco_Ultimate_Guide_to_Manufa cturing.pdf?dtid $=$ oemzzz000233\&ccid $=$ cc000101\&ecid $=2718 \&$ oid $=$ ebkxa 008584

[21] ARC, "Process Automation and the IoT: Yokogawa's Vigilant Plant Approach to the Connected Industrial Enterprise", in "White Paper," ARC Advisory Group February 2015, [Online]. Available: https://webmaterial3.yokogawa.com/arc-iot.pdf.

[22] G. Cline, "The Challenges and Opportunities of OT \& IT Integration", in "Product Innovation and Engineering (PIE) " ABERDEEN 10 October 2017, [Online]. Available: https://www.aberdeen.com/opsproessentials/challenges-opportunities-ot-integration/

[23] N. Papakostas, J. O'Connor and G. Byrne, "Internet of things technologies in manufacturing: Application areas, challenges and outlook", 2016 International Conference on Information Society (i-Society), Dublin, 2016, pp. 126-131. doi: 10.1109/i-Society.2016.7854194

[24] R. Sabella, A. Thuelig, M. C. Carrozza, M. Ippolito. "Industrial Automation enabled by Robotics, Machine Intelligence and $5 \mathrm{G}$ " in "5G and Industrial Automation", ERICSSON, ERICSSON Technology Review 15 February 2018, vol. 2 [Online]. Available: https://www.ericsson.com/assets/local/publications/ericsson-technologyreview/docs/2018/etr 2018-02 robotics web-feb18.pdf.
[25] K. Pittman, "The IIoT in a Nutshell", Advanced Manufacturing, 18 July 2017. Accessed on: 01/05/2019 [Online]. Available: https://www.engineering.com/AdvancedManufacturing/ArticleID/15282/T he-IIoT-in-a-Nutshell.aspx

[26] The Industrial Internet of Things Volume G1: Reference Architecture, IIC:PUB:G1:V1.80:20170131, 2017.

[27] IIoT reference architecture published. 2017. Control Engineering, 64(3), pp. 29.

[28] M. Weyrich and C. Ebert, "Reference Architectures for the Internet of Things", IEEE Software., vol. 33, no. 1, pp. 112-116, 2016.

[29] Reference Architectural Model Industrie 4.0 (RAMI4.0) 2018.

[30] Internet of Things (IoT) - Reference Architecture, ISO/IEC 30141:2018, 2018.

[31] S. Gulliford, "We need to talk about this IoT", Genserv 31 May 2018, [Online]. Available: https://www.gemserv.com/we-need-to-talk-about-thisiot-thing/.

[32] Network \& Information Systems (NIS) Guidance, 2018.

[33] General Data Protection Regulation (GDPR), European Union, 2016.

[34] Department for Digital, Culture Media \& Sport. "Secure by Design: Improving the cyber security of consumer Internet of Things Report", Department for Digital, Culture Media \& Sport, 7 March 2018, [Online]. Available: https://www.gov.uk/government/publications/secure-by-designreport.

[35] The Industrial Internet of Things Volume G4: Security Framework IIC:PUB:G4:V1.0:PB:20160926 2016

[36] B. Barnes, "Cisco Partners with Mazak and MEMEX to connect Machines", 27 October 2015. Accessed on: 31/05/2019[Online]. Available: https://blogs.cisco.com/manufacturing/cisco-partners-with-mazak-andmemex-to-connect-machines

[37] Z. Radovilsky, "Enterprise Resource Planning (ERP)", in The Internet Encyclopaedia, vol. 1, Hossein Bidgoli, Ed.: John Wiley \& Sons, 2004, p. 707.

[38] C. Rathmann, "Industrial Internet of things (IOT) and digital transformation - IoT, Digital Transformation and the role of Enterprise Software" 2017. Available: https://www.ifsworld.com/us/sitecore/medialibrary/assets/2017/09/14/industrial-iot-and-digital-transformation/.

[39] L. Bartevya, "Industry 4.0-Summary Report", 2015, Available: https://www.cenit.com/fileadmin/dam/Corporate/PDFs/2015 5 Experten wissen_E.pdf.

[40] Y. Lav, "Industry 4.0: Harnessing the Power of ERP and MES Integration" 20 July 2017. Available: https://www.industryweek.com/supply-chaintechnology/industry-40-harnessing-power-erp-and-mes-integration

[41] B. Marr, "Big Data: The 5 Vs Everyone Must Know", 6 March 2014. Available: https://www.linkedin.com/pulse/20140306073407-64875646big-data-the-5-vs-everyone-must-know/

[42] P. Fettrman, Manufacturing Metrices: Driving Operational Performance: LNS Research 2018. [Online]. Available: https://www.lnsresearch.com/research-library/researcharticles/manufacturing-metrics. Accessed on 8/5/2019.

[43] OEE Industry Standard 2001.

[44] Cisco, "Kinetic Edge \& Fog Processing Module White Paper", Cisco 2018, Available: https://www.cisco.com/c/dam/en/us/solutions/collateral/internet-ofthings/cisco-kinetic-efm-whitepaper.pdf.

[45] T. Hahn, "Industrial AI applications", in Industrie 4.0 Forum, HANNOVER MESSE, 2019: Big Data Value Association (BDV).

[46] J. Moretti. (2018, 1 February) The technologies empowering Industry 4.0. I4.0 today, Available: http://i40today.com/magazines/

[47] V. Bregulla, "Industrial companies see the massive value of AI in the coming decade", Hewlett Packard Hewlett Packard 25 September 2019, Available: https://www.hpe.com/us/en/newsroom/blogpost/2018/09/industrial-companies-see-the-massive-value-of-ai-in-thecoming-decade.html

[48] L. Veronesi, M. Slowik, K. Prouty, A. Pinder, R. Paquin, N. Mandal, J. Hojlo, S. Ellis "Worldwide Manufacturing 2019 Predictions," in "IDC_FutureScape", October 2018, Available: https://www.idc.com/getdoc.jsp?containerId=EMEA43135818.

[49] P. D.U. Coronado, R. Lynn, W. Louhichi, M. Parto, E.t Wescoat, T. Kurfess", Part data integration in the Shop Floor Digital Twin: Mobile and cloud technologies to enable a manufacturing execution system", Journal of Manufacturing Systems, vol. 48, no. C, p. 9, 2018.

[50] InVima, "Unleashing the Smart Factory", InVMA Limited 2019, Available: https://www.invma.co.uk/unleashing-the-smart-factory.

[51] S. Riley, "Fundamentals of the digital supply chain," Software AG, June 2018, Available: https://info.softwareag.com/Fundamentals-of-SupplyChain-Management.html.

[52] The Growth Company. (2019). Made Smarter. Available: https://www.madesmarter.uk/

[53] R. Sinfield, "Five ways businesses can take advantage of Industry 4.0", IML Group, Connectivity 4IR 14 February 2019, Available: http://www.connectivity4ir.co.uk/article/167393/Five-ways-businessescan-take-advantage-of-Industry-4-0.asp 